\title{
Zur Erneuerung des Geographieunterrichtes in der Schweiz
}

Der Verein Schweizerischer Geographielehrer hat in den Jahren 1972 - 74 eine Reihe von Weiterbildungskursen durchführen können. Finanziell und organisatorisch wurde er dabei von der Schweizerischen Zentralstelle für die berufliche Weiterbildung der Mittelschullehrer in Luzern (Direktor F. Egger) unterstützt, die ihrerseits von der Konferenz der Kantonalen Erziehungsdirektoren getragen wird.

Im Hinblick auf neue Kursziele stellte sich der Vorstand des VSGg die Frage, in welcher Form und in welchem Ausmass die stofflichen Gehalte der bisherigen Kurse im Unterricht in Erscheinung treten. Um dies mindestens teilweise zu erfahren und zur Vermittlung gegenseitiger Anregungen, wurden die Mitglieder aufgefordert, anlässlich der Jahresversammlung Beispiele von Unterrichtserneuerungen und Kursauswertungen, laufende Arbeiten an Lehrplänen und neue Lehrmittel vorzustellen. Wir geben hier die wesentlichen Ergebnisse in Form von Kurzartikeln wieder:

Unterrichtserneuerungen und Kursauswertun gen

Langfristige selbständige Arbeiten am Realgymnasium Zürich.

Am Realgymnasium Zürich läuft seit einiger Zeit ein Reformversuch der Oberstufe (5. -7. Klasse, Sekunda bis Oberprima), der im Herbst 1974 modifiziert worden ist. Danach wird durch linearen Abbau von 3 Semesterstunden ein schulfreier Tag (Mittwoch oder Donnerstag) ermöglicht, der für selbständige grössere Hausarbeiten gedacht ist und zudem den Unterricht störende Veranstaltungen aufzunehmen hat. Die Geographie ist daran im Sommer der 5 . Klasse beteiligt.

Die Organisation ist so gedacht, dass immer drei Fächer gleichzeitig mitmachen. Jedes Fach beschäftigt nach Ermessen einzelne Schüler, kleinere oder grössere Gruppen mit einem oder verschiedenen Themen. Der Abschluss kann in einem mündlichen oder schriftlichen Bericht oder aus einem Vortrag vor der Klasse bestehen. Jeder Lehrer bietet die Schüler in kleinen Gruppen ca. dreimal zu Besprechung und Kontrolle auf, sodass seine Belastung (im Semester) etwa 18 Stunden plus Korrekturen und Vorbereitungen beträgt; entsprechend wird er dafür mit einer Semester- stunde ents chädigt.

Arbeitskonzept: In der Geographie ist geplant, grössere Teilgebiete selbständig erarbeiten zu lassen, z. B. Raumplanung, Ethnographie etc.; dazu dienen Fachlektüren, Sammlungen von Zeitungsausschnitten, Kartierungen, Museums besuche und ähnliches. Der erste Versuch wird im Sommer 1975 stattfinden.

Kernfachkurs-Versuch am Gymnasium BernNeufeld

Seit Herbst 1970 wird am Gymnasium BernNeufeld (Literar- und Realabteilung) im Hinblick auf eine spätere Gymnasialreform ein Versuch mit Kernfachunterricht durchgeführt. Er besteht darin, dass im letzten Jahr vor der Matur die Schüler in Gruppen von 6-15 Teilnehmern in 2 frei gewählten Fächern einen vertieften Unterricht mit je 5 Wochenstunden erhalten. Die normale Stundentafel wird dagegen in den meisten Fächern entsprechend gekürzt. An der Durchführung der Maturitätsprüfungen ändert sich nichts: im reduzierten Normalunterricht muss das obligatorische Maturitäts pensum erreicht werden und auch die Notenund Promotionsordnung bleibt im allgemeinen unverändert.

Organisation: Als Kernfächer können praktisch alle Schulfächer gewählt werden, wobei minde stens eines der gewähltenKernfächer Maturitäts fach sein muss. Dem Lehrer werden für die 5 wöchentlichen Kernfachstunden der Mehrbelastung wegen 7 Stunden angerechnet. Für das Fach Geographie ergeben sich nun sehr schöne Möglichkeiten. Unser Normalunterricht wird bis Mitte Prima, d. h. ein Jahr vor der Matur geführt, und der Kernfachkurs schliesst dann direkt an. Auf der Grundlage des Normalunterrichts kann sich der Kernfachunterricht auf wenige Problemkreise beschränken. Wir haben bis jetzt folgendes Arbeitskonzept verfolgt:

- Jeder Schüler verarbeitet während des Kur ses ein Hauptthema, das inhaltlich und formal sauber dargestellt werden muss.

- Wenn immer möglich - die Schüler haben ein Mitspracherecht bei Stoffauswahl und Unterrichtsgestaltung - werden diese Hauptarbeiten um ein Generalthema gruppiert. Im letzten Jahr z. B. wurden verschiedene Aspekte der Gemeinde Lenk im Simmental bearbeitet: Fremdenverkehr, Berglandwirtschaft, 
Abwanderung, Ortsplanung, Klima. Aehnlich wurde in einem früheren Kurs die Gemeinde Moosseedorf, eine typische Wachstumsgemeinde im Bereiche eines Autobahnanschlusses, analysiert.

- Neben dieser Hauptarbeit werden kleinere Themen behandelt, vor allem zur Auflockerung: Luftbildbesprechungen, Diskussionen über Wirtschaftsfragen, alte Karten usw. Die Schülergruppen sind oft recht inhomogen, weil unterschiedliche Motive zum Besuch eines Kurses führen, z. B.

Interesse am $\mathrm{Fach}$,

Vorbereitung auf späteres Gg-Studium, Auskneifen vor andern Fächern.

Erfahrungen: Das Niveau, das dem Kurs gegeben wird und die Dosierung des Leistungsdrucks beeinflusst die Zusammensetzung der Gruppe. Der Kernfachkurs ermöglicht eine Vertiefung des Unterrichts (Stoff und Methoden), fördert die Teamarbeit (Kleingruppen), gestattet auf der Grundlage des Normalunterrichts eine exemplarische Stoffauswahl und entwickelt das sprachliche Ausdrucksvermögen.

Unser Kernfachsystem ist nicht unbestritten. Einige Fächer, in denen kein Kurs zustande kommt und andere, in denen der Maturstoff in der reduzierten Stundentafel schwer zu bewältigen ist (z. B. Math.) stellen ihn in Frage. Aber die überwiegende Mehrheit der Lehrerschaft und vor allem fast alle Schüler stehen - trotz erheblicher Mehrbelastung auf beiden Seiten positiv zu diesem Versuch.

Arbeitswochen für Ortsplanung am Freien Gymnasium Zürich

Ueber Arbeitswochen für Ortsplanung siehe Wyder S., 1975: Geographica Helvetica Heft 1/ 1975, S. 39-41.

Mitarbeit an der Regionalplanung Sarneraatal durch das Kollegium Sarnen (Kantonsschule Obwalden)

Die Kantonsschule Obwalden in Sarnen konnte sich während des Schuljahres 1973/74 am Regionalplanungsprojekt:" Entwicklungskonzept Sarneraatal" beteiligen. Dadurch wurden einige Abteilungen der mittleren und oberen Gymnasialstufe und den Handelsklassen mit Raumplanungsfragen konfrontiert. Nebst dem Kontakt mit diversen Arbeitstechniken, wurden folgende Ziele angestrebt:
- die Schüler für Zukunftsprobleme besser motivieren und engagieren,

- die persönliche Meinungsbildung fördern,

- die theoretisch angeeigneten Erkenntnisse vertiefen und

- die selbständige und schöpferische Tätigkeit steigern.

Organisation: Anlässlich einer Orientierungsversammlung wurde der Lehrkörper vom damaligen Präsidenten des kantonalen Wirtschaftsrates (Dr. V. Girtanner) und einem Vertreter des beauftragten Planungsbüros über die Wirtschaftslage Obwaldens und das angestrebte Entwicklungskonzept orientiert. Nach einer Bedenkzeit wurden vor Beginn des Schulj ahres $1973 / 74$ in einer zweiten Versammlung die Themen erweitert, differenziert und bestimmt. Durchführung: Folgende Themen wurden in Einzel- und Gruppenarbeiten behandelt:

- das Problem der definitiven Abwanderung: bevorzugte Räume und Beweggründe zur Abwanderung,

- Ursachen der Pendelwanderung und

- Versorgung mit Konsumgütern und Dienstleistungen.

Eine Eignungsstudie war ein weiterer interessanter Versuch. In Form einer eintägigen Exkursion - und im Beisein von Mitarbeitern des beauftragten Planungsbüros - galt es, die Eignung ausgewählter Testregionen des Sarneraatales in bezug auf Landwirtschaft, Industrie, Fremdenverkehr und Dienstleistungen zu überprüfen. Dabei wurden jeweils naturräumliche, verkehrstechnische und soziologische Eignungs kriterien benützt, die ihrerseits mit sachlich abgewogener Punktezahl gewichtet wurden, so dass man abschliessend zur Bearbeitung von Leitbildern schreiten konnte.

Ein drittes "Uebungsfeld" lieferten die Planungsberichte. Zusammenfassungen einzelner Kapitel und kritische Stellungsnahmen versuchten, das objektive Ürteil zu fördern und einen abgerundeten Ueberblick zu verschaffen.

Erfahrungen: Die einleitend formulierten Intentionen konnten weitgehend verwirklicht werden. Zugleich förderte der gesamte Arbeitsablauf das gegenseitige Verständnis unter den Schülern, er aktivierte das Lehrer-Schüler-Verhältnis und förderte schliesslich auch die Kooperation der Lehrer. Im Verlaufe der Planungstätigkeit sind aber einige Probleme aufgetaucht, die bei künf- 
tigen, ähnlichen Vorgängen überdenkt und geändert werden müssen:

- Einzelne Aufgabenstellungen verlangten eine langwierige Aufbereitung und Erarbeitung des Urmaterials. Dadurch wurde es schwierig, ein Nahziel zu verfolgen, und die Motivation liess sichtlich nach. Deshalb ist es wichtig, gut definierte und interessante Teilziele einzubauen.

- Es ist unbedingt erforderlich, vor jeder grösseren Enquete ein Probeverfahren einzuleiten, um die örtlichen Verhältnisse zu berücksichtigen und Unannehmlichkeiten rechtzeitig auszuschalten.

- Vom psychologischen wie auch methodischen und materiell-technischen Standpunkte aus sind erst durch Einführung von Intensivtagen (oder Studienwochen) optimale Arbeitsbedingungen geschaffen.

Literatur: von Wyl E., 1974: Kollegium Sarnen und Regionalplanung Sarneraatal. Jahresbericht 1973/74 der Kantonsschule Obwalden, Kollegium Sarnen, S. 77-88.

\section{Ethnologie im Unterricht}

Wer reist, wird früher oder später auf Hochoder Primitivkulturen stossen, sei dies in Lateinamerika, Afrika, Indien oder anderswo. Besonders der Geograph, von seinem Fach her geschult für die Beobachtung und den Vergleich von kulturellen Zusammenhängen, wird dabei häufig immer mehr in den Bann der Ethnologie gezogen. Leider unterbleibt aber oft die Behandlung solcher Probleme in den eigenen Klassen aus Zeitgründen. Dabei lässt sich im Unter richt feststellen, dass besonders in den oberen Klassen ein reges Interesse für ethnologische Fragen vorhanden ist, sofern ein geeignetes Anschauungsmaterial (Dias, Film, Sammlungsgegenstände) den Schülern erläutert werden kann. Zu empfehlen wäre der vermehrte Besuch von guten Ausstellungen (z. B. Rietberg Museum in Zürich und andere), wenn möglich mit Arbeits gruppen. Eine sachkundige Führung kann auf Wunsch meistens mit der betreffenden Leitung vereinbart werden. Im Zeitalter der sich erschöpfenden Rohstoffe kann uns das Studium von Hoch- und Primitivkulturen, die teils während Jahrtausenden ohne - oder mit teilweiser Uebernutzung ihres Lebensraumes bestanden haben, wertvolle Einsichten vermitteln. Es wäre zu hoffen und liegt auch im Interesse einer mọglichst breiten Schulbildung, dass die Ethnologie wieder vermehrt Berücksichtigung findet und nicht mehr abschätzig als "Reliktgeographie" bezeichnet wird.

Als konkretes Ziel könnte gelten, mit der Schweizerischen Ethnologischen Gesellschaft eine Zusammenstellung zu erarbeiten, aus der für Mittelschul- und Volksschullehrer ersichtlich wäre, zu welchen Unterrichtsstoffen sie im einen oder andern Museum eine permanente ethnologische Ausstellung fänden.

Lehrerfortbildung im Schweizerischen Alpinen Museum (Bern)

Im Januar und Mai 1974 fanden im Schweizerischen Alpinen Museum Bern zwei Lehrerfortbildungskurse statt, die unter dem Titel "Beiträge zur Heimatkunde des Kantons Bern anhand des Museumsgutes" von der Fortbildungs zentrale des Bernischen Lehrervereins ausgeschrieben worden waren. Jeder Kurs umfasste drei Spätnachmittage und widmete sich je drei Sammlungsbereichen des Museums.

Die umfangreiche Reliefsammlung lieferte ergiebiges Anschauungsmaterial zu folgenden Bereichen: Geographische Raumübersicht, Gelände und Karte, geologische und tektonische Kammerung, morphologische Einheiten, Siedlungsund Verkehrsstruktur. Anhand der Kartensammlung konnte die Entwicklung der Kartographie seit dem Ende des 15. Jh. verfolgt werden. Folgende Probleme wurden gezielt beobachtet: Orientierung der Karten, Reliefdarstellung, Grundrissituation, Generalisierung und Signaturen, schmückendes und belehrendes Beiwerk am Kartenrand und zum Ortsnamenbestand. Im dritten Teil befasste man sich mit den landeskundlichen Aussagen in der Reiseliteratur des 18. und 19. Jh. Für den Unterricht sind hier zahlreiche und höchst interessante Angaben über Verkehrswege, Reisezeiten, Transportmittel, Unterkunftsverhältnisse und Siedlungszustände herauszuholen.

Gemischte Arbeitsgruppen aus Museums- und Schulleuten könnten unmittelbar nutzbringende Arbeit leisten, da die materiellen Grundlagen bereits bestehen. Mit bescheidenem finanziellem Aufwand wäre ein sehr grosser Nutzeffekt zu erzielen.

(Fortsetzung folgt im Heft 3/1975) 